\title{
Dynamic Modeling of Stick Slip Motion in an Untethered Magnetic Micro-Robot
}

\author{
Chytra Pawashe*, Steven Floyd*, and Metin Sitti
}

\begin{abstract}
This work presents the dynamic modeling of an untethered electromagnetically actuated magnetic micro-robot, and compares computer simulations to experimental results. The micro-robot, which is composed of neodymium-iron-boron with dimensions $250 \mu m \times 130 \mu m \times 100 \mu m$, is actuated by a system of 5 macro-scale electromagnets. Periodic magnetic fields are created using two different control methods, which induce stick-slip motion in the micro-robot. The effects of model parameter variations on micro-robot velocity are explored and discussed. Micro-robot stick-slip motion is accurately captured in simulation. Velocity trends of the micro-robot on a silicon surface as a function of magnetic field oscillation frequency and magnetic field strength are also captured. Mismatch between simulation and reality is discussed.
\end{abstract}

\section{INTRODUCTION}

The fundamental challenge with decreasing robot size below the centimeter scale is providing power and actuation to the robot. Most current micro-robots rely on external actuation and/or power to function, and usually have further limitations as well. These limitations include restrictions such as requiring tethers [1]-[3], a fluid environment [4]-[7], or a specialized operating surface [8]-[11]. Further, though many miniature robots exist on the centimeter or millimeter scale, true micron-scale robots, with all characteristic lengths on the order of tens to hundreds of microns, are still quite rare [4], [5], [9], [11], [12].

The micro-robot presented in this and in earlier work [13] utilizes magnetic torque provided by large-scale electromagnets to achieve its motion and does not require any physical tethers or an on-board power source. Controlled by dynamically adjusting magnetic fields, it does not need a patterned work surface or electrostatic coupling to a subsurface. Additionally, while the robot does not require a fluid medium to operate, it can move and perform tasks in a fluid environment. Some limitations of this design are that the working surface cannot be composed of a ferromagnetic or strongly diamagnetic material, nor can the surface itself be magnetized, and the robot must remain within the working volume of the electromagnets.

In this paper, we present a comprehensive dynamic model of the magnetic micro-robot and its interactions with the magnetic field and the silicon surface on which it operates. Such a model is necessary to understand the nature of the

* Equally contributing co-first authors

C. Pawashe and S. Floyd are with the Department of Mechanical Engineering, Carnegie Mellon University, Pittsburgh, PA 15213, USA

[csp, srfloyd] Candrew. cmu.edu

M. Sitti is with the Department of Mechanical Engineering and Robotics Institute, Carnegie Mellon University, Pittsburgh, PA 15213, USA sittiecmu.edu robot's motion, and can be used as a tool for future optimization and control. With an accurate model, the number of necessary experiments can be greatly reduced, and time consuming or difficult tests can be performed in computer simulation, allowing for quick parameter optimization.

Micro-robots that do not rely on specialized surfaces for power delivery and control are a vital step toward advancing the field of micro-robotics, which is filled with many potential applications. Examples include micro-manipulation of micro-components, and micro-assembly and fabrication of hybrid micro-systems [8], [10]. Tetherless micro-robots with appropriate tools can be used for micro-scale measurement and surface inspection [1]. More advanced microrobots could even be used for undetectable surveillance as micro-unmanned vehicles, or as micro-surgeons in medical applications inside living bodies [2].

\section{EXPERIMENTAL SETUP}

Five independent electromagnetic coils were constructed large enough to enclose a cube $10 \mathrm{~cm}$ on a side, which contains the working volume. Of these coils, four were placed upright to control the direction and gradient of the horizontal magnetic field, and one is placed below the work plane to control electromagnetic clamping, as seen in Fig. 1. Within the tolerances of machining, the coils were constructed to be identical, with the same dimensions, wire gauge, and number of turns of the wire. Imaging of the the magnetic micro-robot is accomplished with a CCD camera connected to a variable magnification microscope lens. For high-framerate video, a high speed camera (Phantom V7.0) and an additional microscope lens was placed horizontally inside one of the four upright magnets to achieve a side view of the micro-robot during actuation. Parameters for each of the electromagnets are provided in Table I. Control of the electromagnetic coils is performed by a $\mathrm{PC}$ with a data acquisition system at a control bandwidth of $1 \mathrm{kHz}$. The coils are powered by custom-made electronic amplifiers, controlled by the PC.

\section{RoBOT FABRICATION}

The micro-robot used in these experiments was made of neodymium-iron-boron $(\mathrm{NdFeB})$, a hard magnetic material. To create the robot, a magnetized piece of $\mathrm{NdFeB}$ was cut using a laser machining system (NewWave LaserMill). First, the $\mathrm{NdFeB}$ was cut parallel to the direction of magnetization, making planar slices approximately $100 \mu \mathrm{m}$ thick. These slices were then laid flat so that the magnetization of the slice was in a horizontal plane. Robots were then cut from 


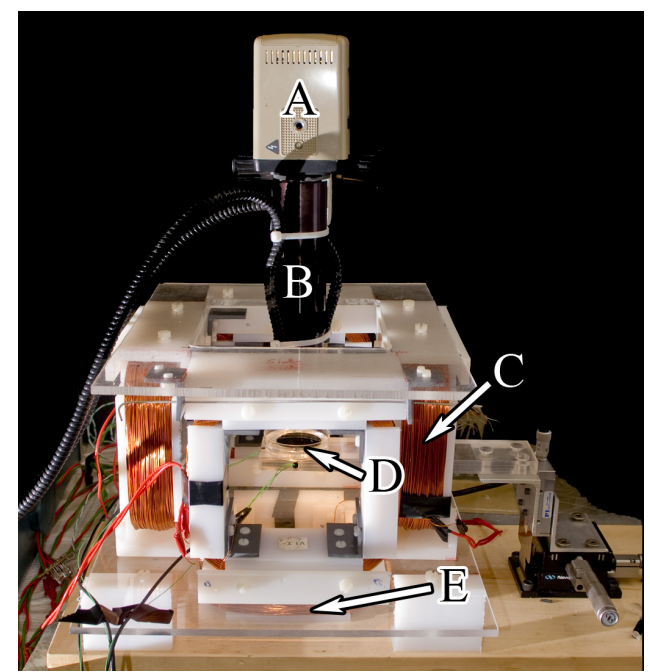

Fig. 1. Photograph of the electromagnetic coil setup, where (A) is the camera, (B) is the microscope lens, (C) is one of four horizontal coils that move the micro-robot within the plane, (D) is the wafer where the microrobot resides, and $(\mathrm{E})$ is the clamping coil beneath the wafer that holds the micro-robot to the surface.

these slices such that the magnetization vector was pointing towards the front of the robot. High translational speeds, small laser spot size, and low cutting depths per pass were employed to minimize local demagnetization due to heating by the laser.

\section{Modeling}

A computer simulation was created to model the behavior of the micro-robot as it interacts with the silicon surface it moves upon, and how it is affected by the magnetic fields created by the five macro-scale electromagnets over time. Being made of hard magnetic material, the magnetic field does not affect the robot's internal magnetization. Furthermore, the small size of the robot implies that the magnetic field it creates does not significantly affect the electromagnets.

\section{A. Magnetic Field}

The magnetic field produced is determined by the current, $I$, through the electromagnetic coil. It is a function of the voltage across the coil, $V$, which is a control input in the

\begin{tabular}{|c||c||c|}
\hline Description & Value & Units \\
\hline Coil Resistance $(R)$ & 10.0 & $\Omega$ \\
\hline Inductance $\left(L_{i}\right)$ & 70 & $m H$ \\
\hline Inner length & 0.120 & $m$ \\
\hline Outer length & 0.157 & $m$ \\
\hline Number of Turns $\left(N_{t}\right)$ & 510 & - \\
\hline Effective Length & 0.1385 & $m$ \\
\hline Distance From Center & .099 & $m$ \\
\hline Maximum Field at Center & 6.5 & $m T$ \\
\hline Maximum Gradient at Center & 149 & $m T / m$ \\
\hline
\end{tabular}

TABLE I

Electromagnet PRoperties simulation, and the resistance and inductance of the coil. Resistance and inductance were measured experimentally, and their values are presented in Table I. Using these values, the differential equation for the current through an inductor is incorporated in the dynamic simulation:

$$
\frac{d I}{d t}=\frac{-R}{L_{i}} I+\frac{1}{L_{i}} V
$$

This current is used to determine the magnetic field produced by each of the coils.

Inside of the control volume, the principle of superposition is valid for determining the magnetic field at a point in space. Hence, the contributions from all five electromagnets can be determined separately and then added together. To determine the contribution of each electromagnet, one must apply the Biot-Savart law for each square turn coil:

$$
\vec{B}(\vec{X})=\frac{\mu_{0} N_{t} I}{4 \pi} \oint_{S} \frac{\overrightarrow{d l^{\prime}} \times \vec{a}_{R}}{R^{2}}
$$

where $\vec{B}(\vec{X})$ is the magnetic field at the robot's position $\vec{X}=x \vec{e}_{x}+y \vec{e}_{y}+z \vec{e}_{z}, \mu_{0}$ is the permeability of free space $\left(4 \pi \times 10^{-7}\right), \overrightarrow{d l^{\prime}}$ is an infinitesimal line segment along the direction of integration, $\vec{a}_{R}$ is the unit vector from the line segment to the point in space of interest, and $R$ is the distance from the line segment to the space of interest.

For a square turn coil, this contour integral simplifies into four line integrals, the definite integral of which exists and can be evaluated at the end points [14]. For the $x$-directed coils, the primary field in $x$ and the fringe fields in $y$ and $z$ can be determined at any point in space by evaluating the following [15]:

$$
\begin{aligned}
& R= \sqrt{(x-c)^{2}+\left(y-y_{j}\right)^{2}+\left(z-z_{i}\right)^{2}} \\
& B_{x}= \frac{\mu_{0} N_{t} I}{4 \pi} \sum_{i=1}^{2} \sum_{j=1}^{2}(-1)^{i+j} * \frac{\left(z-z_{i}\right)\left(y-y_{j}\right)}{R} \\
& *\left[\frac{1}{(x-c)^{2}+\left(y-y_{j}\right)^{2}}+\frac{1}{(x-c)^{2}+\left(z-z_{i}\right)^{2}}\right] \\
& B_{y}= \frac{\mu_{0} N_{t} I}{4 \pi} \sum_{i=1}^{2} \sum_{j=1}^{2}(-1)^{i+j+1} \\
& *\left(\frac{\left(z-z_{i}\right)(x-c)}{R} *\left[\frac{1}{(x-c)^{2}+\left(y-y_{j}\right)^{2}}\right]\right) \\
& B_{z}= \frac{\mu_{0} N_{t} I}{4 \pi} \sum_{i=1}^{2} \sum_{j=1}^{2}(-1)^{i+j+1} \\
& *\left(\frac{(x-c)\left(y-y_{j}\right)}{R} *\left[\frac{1}{(x-c)^{2}+\left(z-z_{i}\right)^{2}}\right]\right) \\
& z=[a,-a], y=[a,-a]
\end{aligned}
$$

where $B_{i}$ is the magnetic field in the $i$ direction, $a$ is half the effective length of the electromagnet, and $c$ is \pm the distance from the center, where the \pm is evaluated based upon the location of the coil of interest (i.e. plus for the coil in the positive $x$ or positive $y$-directions). Similar equations are used for the $y$-directed and clamping coils.

This derivation is for a concentrated electromagnet, i.e. all the current carrying wires can be described by a single line with zero thickness. This assumption is found to be accurate to within $2.2 \%$ because the travel distances are small in comparison to the magnetic coils, and the distribution of wires within each electromagnet is small compared to the 
size of the magnet. As a result, an effective length must be used instead of the actual inner or outer length; these values are listed in Table I.

\section{B. Magnetic Forces}

Within a magnetic field any magnetized object, in this case the micro-robot, will experience both a torque and a force. This magnetic torque is proportional to the magnetic field strength, and acts in a direction to bring the internal magnetization of the object into alignment with the field. The magnetic force is proportional to the gradient of the magnetic field, and acts to move the object to a local maximum. The equations that govern these interactions are:

$$
\begin{gathered}
\overrightarrow{T_{m}}=V_{m} \vec{M} \times \vec{B}(\vec{X}) \\
\overrightarrow{F_{m}}=V_{m}(\vec{M} \bullet \nabla) \vec{B}(\vec{X})
\end{gathered}
$$

where $\overrightarrow{T_{m}}$ is the torque the robot experiences, $V_{m}$ is the volume of the robot, $\vec{M}$ is the magnetization of the robot (assumed to be uniform) [7], [16].

The gradient is determined analytically by taking the derivatives of (4-6) in each direction, yielding nine terms. Torque is determined by finding the magnetic field at the center of the robot's body. It was found that for a cube 2.5 $\mathrm{cm}$ on a side in the center of the working volume, fringing fields were less than $2.5 \%$ of the primary field for each coil individually, and the maximum force and torque that can be exerted on the micro-robot are $240 \mathrm{nN}$ and $10.5 \mu \mathrm{N} \cdot \mathrm{mm}$, respectively.

\section{Magnetic Micro-Robot}

In modeling the micro-robot, we assume it is an isotropic rectilinear solid with the properties tabulated in Table II.

\begin{tabular}{|c||c||c|}
\hline Description & Value & Units \\
\hline Length $(L)$ & 250 & $\mu \mathrm{m}$ \\
\hline Width $(W)$ & 130 & $\mu \mathrm{m}$ \\
\hline Height $(H)$ & 100 & $\mu \mathrm{m}$ \\
\hline Density $(\rho)$ & 7400 & $\mathrm{~kg} / \mathrm{m}^{3}$ \\
\hline Mass $(m)$ & 25.6 & $\mathrm{ng}$ \\
\hline Weight $(m g)$ & 251 & $\mathrm{nN}$ \\
\hline Magnetization $(M)$ & $5 \times 10^{5}$ & $\mathrm{~A} / \mathrm{m}$ \\
\hline
\end{tabular}

TABLE II

MAgnetic RoBot Properties

From experimental high-speed video of the micro-robot, it appears to exhibit a stick-slip motion when actuated by a pulsed magnetic field. During motion, the robot moves by rocking forward and backward around a steady state angle. Motion occurs when the contact point between the robot and the silicon surface slips. This occurs either when the robot "falls" while rocking down, or "jumps" while rocking up. Several images from a high speed video at 200 frames per second (fps) are presented in Fig. 2(a-d) to illustrate this motion. A video can be found online at [17]. We attempt to model this behavior in a computer simulation.

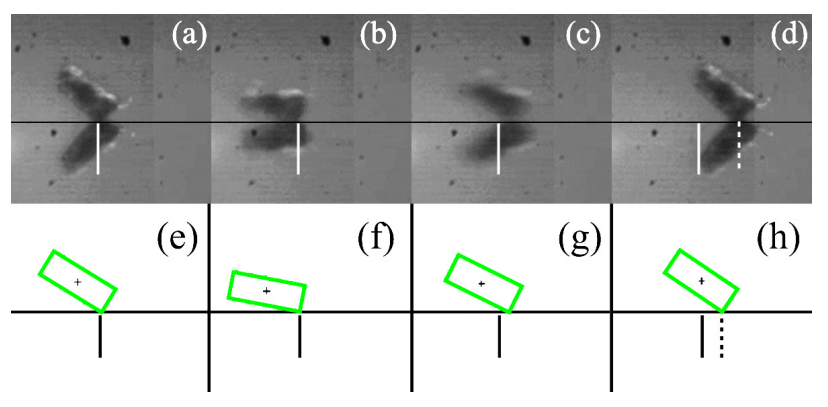

Fig. 2. Stick-slip motion of the micro-robot observed with a high speed camera, compared to simulated results. (a) The robot is initially at its steady state angle, and the point of contact is highlighted by a solid white line. (b) When the magnetic field changes, the robot rocks downward, changing its angle relative to the silicon wafer. (c) During the next upswing, the robot slides forward. The former contact point is highlighted with a solid white line. (d) After slipping forward, the robot assumes its steady state angle again at a new position, shown by a dashed white line. Analogous steps are performed in simulation in images (e) through (h).

To simulate the dynamics of the magnetic micro-robot, we restrict modeling to a side-view of the robot in the $x-z$ plane, shown in Fig. 3. The robot has a center of mass (COM) at $\vec{X}$, an orientation angle $\theta$ from the ground, a distance $r$ from its COM to a corner, and an angle $\phi$ determined from geometry. The robot experiences external forces, including its weight, $m g$, a normal force from the surface, $N$, an adhesive force to the surface, $F_{a d h}$, an $x$-directed externally applied magnetic force, $F_{x}$, a $z$-directed externally applied magnetic force, $F_{z}$, a linear damping force in the $x$-direction, $L_{x}$, a linear damping force in the $z$-direction, $L_{z}$, an externally applied magnetic torque, $T_{y}$, a rotational damping torque, $D_{y}$, and a Coulomb sliding friction force $F_{f} . F_{f}$ depends on $N$, the sliding friction coefficient $\mu$, and the velocity of the contact point, $\frac{d P_{x}}{d t}$, where $\left(P_{x}, P_{z}\right)$ is the bottom-most point on the micro-robot (nominally in contact with the surface). Using these forces, we develop the dynamic relations:

$$
\begin{aligned}
m \ddot{x}= & F_{x}-F_{f}-L_{x} \\
m \ddot{z}= & F_{z}-m g+N-F_{a d h}-L_{z} \\
J \ddot{\theta}= & T_{y}+\left(F_{f}\right) r \sin (\theta+\phi) \\
& -\left(N-F_{a d h}\right) r \cos (\theta+\phi)-D_{y}
\end{aligned}
$$

where $J$ is the polar moment of inertia of the robot, calculated as $J=m\left(H^{2}+L^{2}\right) / 12$.

The robot is first assumed pinned to the surface at $\left(P_{x}, P_{y}\right)$, where $0<\theta<\frac{\pi}{2}$. This gives the following additional equations:

$$
\begin{array}{r}
x=P_{x}-r \cos (\theta+\phi) \\
\ddot{x}=\ddot{P}_{x}+r \ddot{\theta} \sin (\theta+\phi)-r \dot{\theta}^{2} \cos (\theta+\phi) \\
z=P_{z}+r \sin (\theta+\phi) \\
\ddot{z}=\ddot{P}_{z}+r \ddot{\theta} \cos (\theta+\phi)-r \dot{\theta}^{2} \sin (\theta+\phi)
\end{array}
$$

To solve equations (10-14), we realize that there are 7 unknown quantities $\left(N, \ddot{\theta}, \ddot{x}, \ddot{z}, \ddot{P}_{x}, \ddot{P}_{z}, F_{f}\right)$ for 5 equations, indicating an under-defined system. As the stick-slip motion in this system is similar to the case outlined by Painlevé's paradox, we resolve the paradox by taking the friction force, 


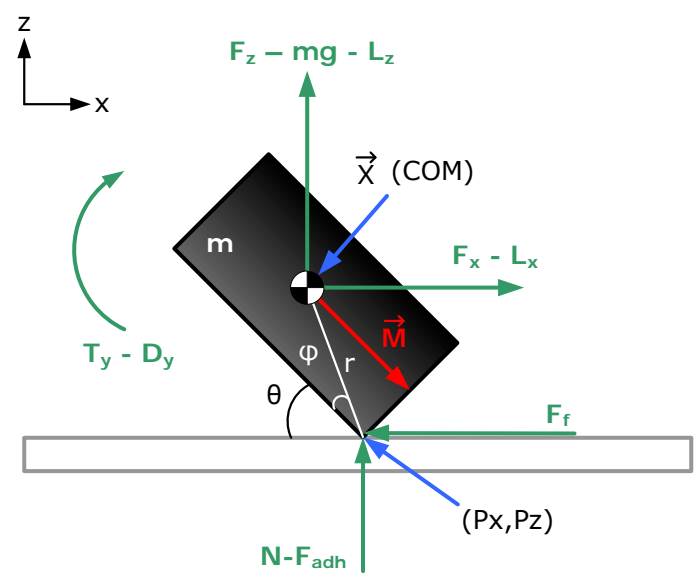

Fig. 3. Schematic of a rectangular magnetic micro-robot with applied external forces and torques. Magnetization vector denoted by $\vec{M}$.

$F_{f}$, as an unknown value (as opposed to setting $F_{f}=\mu N$ ) [18]. Using the pinned assumption, we can set $\ddot{P}_{x}=\ddot{P}_{z}=0$; then, equations (10-14) are solved directly. There are three possible types of solution that can occur during each time step:

1. The solution results in $N<0$ (an impossible case). This implies that the pinned assumption was false, and the micro-robot has broken contact with the surface. Equations (10-12) are resolved using $N=0$ and $F_{f}=0$.

2. The solution results in $F_{f}>F_{f \max }$, where $F_{f \max }=$ $\mu \cdot N$. This also implies that the pinned assumption was false, and the point of contact is slipping; thus the robot is translating in addition to rocking. Equations (10-14) are resolved using $F_{f}=F_{f \max }$ and $\ddot{P}_{x}$ left as an unknown.

3. All of the variables being solved for are within physically reasonable bounds. The robot is in contact with the surface at the pinned location and is rocking in place.

When a satisfactory solution is reached for each time step, the solutions for orientation and location are used as initial conditions in the next solution step.

\section{Simulation Results}

To simulate the micro-robot, a $5^{t h}$ ordered Runge-Kutta solver is used to solve the time-dependent system. A magnetic pulsing signal is given as a voltage waveform, and equation (1) is solved for the currents. With given initial conditions, equations (1-9) are used to determine the magnetic field forces, and equations (10-14) are solved for the three position states of the micro-robot: $x, z$, and $\theta$. The results of the simulation are displayed in Figure 2(e-h), where the simulated micro-robot exhibits stick-slip motion, agreeing with experiment.

From previous work [13], two different types of magnetic actuation can be used to move the magnetic micro-robot in a reliable fashion. The first is In Plane Pulsing (IPP), where the magnetic field within the plane of motion is varied, while the clamping magnetic field is held constant. The second method is Out of Plane Pulsing (OPP), where the magnetic field within the plane of motion is held constant, but the clamping magnetic field is varied. Since a DC magnetic field will not translate a micro-robot due to high static friction, these periodic excitation methods are necessary to induce stick-slip translation.

\section{A. In Plane Pulsing}

For IPP control, the voltages across the clamping electromagnet and one in-plane electromagnet (in the direction of robot motion) are slowly ramped up. This causes the robot to orient in the desired direction while remaining locked down on the silicon surface. Next, the in-plane electromagnet is pulsed using a sawtooth waveform at a higher voltage, varying the horizontal, or $x$-directed magnetic field. The magnetic fields generated in the simulation from IPP control are shown in Fig. 4.

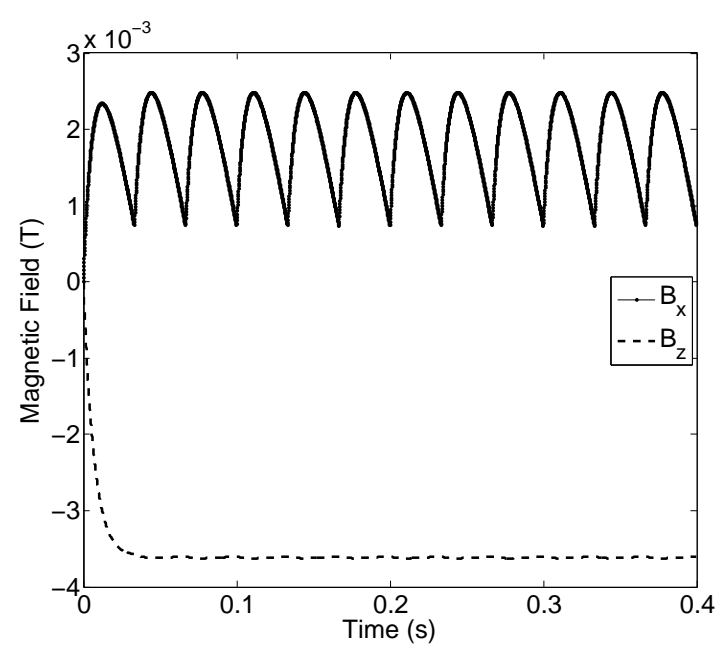

Fig. 4. Simulation of the $x$ and $z$-directed magnetic fields generated by IPP control at a maximum pulsing magnetic field strength of $2.5 \mathrm{mT}$ with a pulsing frequency of $30 \mathrm{~Hz}$ in simulation.

This alternating in-plane magnetic field causes the robot to rock downward ( $\theta$ decreases) as the field is increased, and rock upward when the field is decreased. The robot translates by slipping each time it rocks downward, which agrees with experimentally observed behavior. After reaching its target destination, the voltage across the in-plane electromagnet is ramped down, leaving only the clamping electromagnet active. A simulation of this motion is presented in Fig. 5.

\section{B. Out of Plane Pulsing}

In OPP control, the voltages across the clamping electromagnet and one in-plane electromagnet (in the direction of robot motion) are slowly ramped up, like in IPP control. After orienting the robot, however, the voltage across the clamping electromagnet is varied using a sawtooth waveform, while the horizontal magnetic field is held constant. The magnetic fields generated in the simulation from OPP control are shown in Fig. 6.

By alternating the out-of-plane magnetic field, the robot tends to rock upward ( $\theta$ increases) as the clamping field is increased, and rock downward when the field is decreased; 


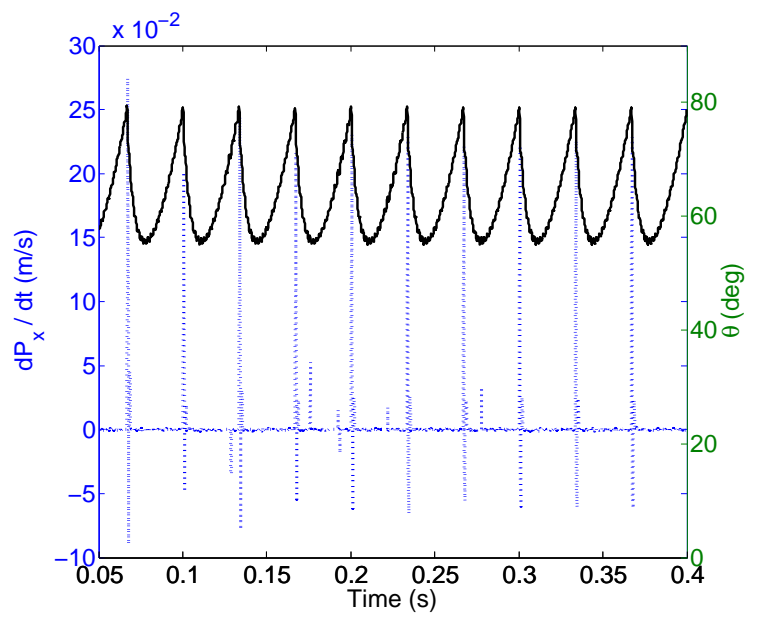

Fig. 5. Simulation of the robot's angle $\theta$ (solid line) and the $x$-direction velocity of the contact point, $\frac{d P_{x}}{d t}$ (dotted line) in a simulation of IPP control. The robot slides over the surface during the downstroke, when $\theta$ is decreasing.

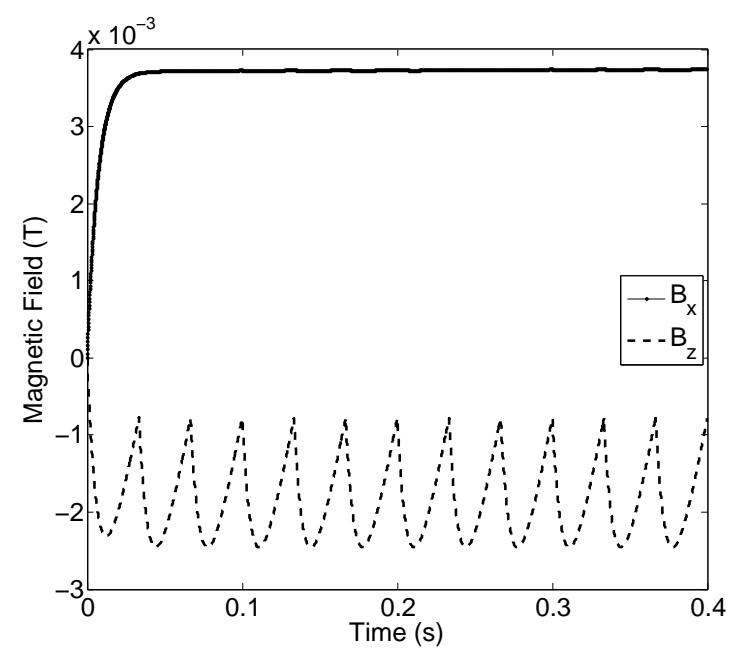

Fig. 6. Simulation of the $x$ and $z$-directed magnetic fields generated by OPP control at a maximum pulsing magnetic field strength of $2.5 \mathrm{mT}$ with a pulsing frequency of $30 \mathrm{~Hz}$ in simulation.

this is an opposite effect when compared to IPP control. In this case, the robot translates by slipping each time it rocks upward, which agrees with experimentally observed behavior, and is explicitly shown in Fig. 2. After reaching its target destination, the voltage across the in-plane electromagnet is ramped down, leaving only the clamping electromagnet active. A simulation of this motion is presented in Fig. 7.

\section{Matching Model Parameters}

In order to accurately model the physical system, several parameters had to be determined empirically. As with any empirical determination, there exists the possibility of error.

\section{A. Friction}

The Coulomb sliding friction coefficient between the robot and the surface, $\mu$, was determined by using a load-cell to

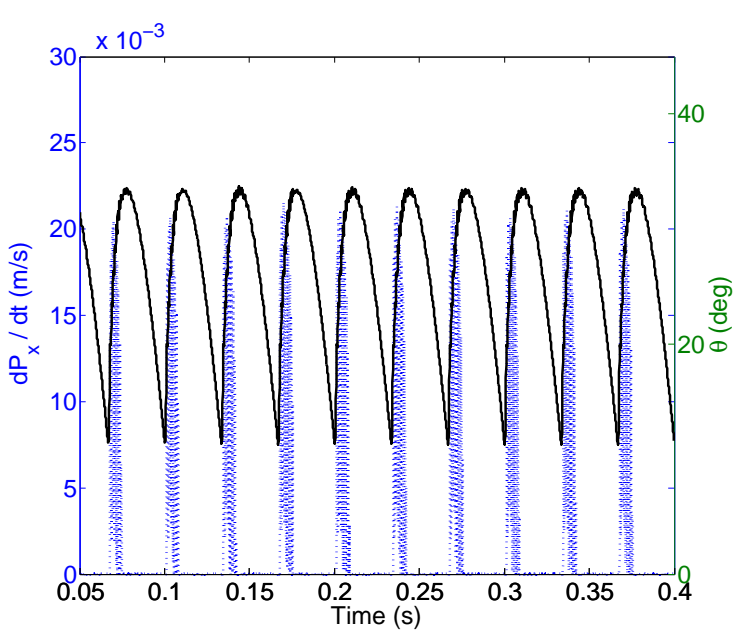

Fig. 7. Simulation of the robot's angle $\theta$ (solid line) and the $x$-direction velocity of the contact point, $\frac{d P_{x}}{d t}$ (dotted line) in a simulation of OPP control. The robot slides over the surface during the upstroke, when $\theta$ is increasing.

measure the force required to slide a bulk NdFeB micromagnet with a known downward load across a Si surface. From this, the friction coefficients between $\mathrm{NdFeB}$ and $\mathrm{Si}$ were found to be $\mu_{k}=0.145$ for kinetic, and $\mu_{s}=0.2$ for static. If $\left|\frac{d P_{x}}{d t}\right|>0, \mu=\mu_{k}$, otherwise $\mu=\mu_{s}$.

Noting that the kinetic friction coefficient is approximately $70 \%$ the static friction coefficient, the effect of varying $\mu$ was explored in simulation while maintaining this ratio, as shown in Fig. 8. At low friction coefficients, the micro-robot dominantly slides; motion due to stick-slip behavior is less dominant as stick-slip motion requires friction. Speeds are higher for OPP control, because the in-plane coil is always on. As the friction coefficient increases, stick-slip behavior becomes more dominant and results in very similar velocity profiles for the two control methods between $\mu_{s}=0.2$ and $\mu_{s}=1.0$. At friction coefficients $\mu_{s}>1.0$, IPP results in higher velocities. This is due to the reduced normal force which arises during IPP downstroke motion, shown in Fig. 5 , as opposed to the increased normal force which arises in upstroke motion for OPP, shown in Fig. 7.

\section{B. Adhesion}

Adhesive forces between the surface and the micro-robot are present due to stiction effects that become significant at the micro-scale, such as capillary and van der Waals forces [19]; these are lumped together into one force $F_{a d h}$. We estimate this force to be $F_{a d h}=0.22 \mu N$, or about $90 \%$ of the micro-robot's weight. To determine this, a micro-robot was placed on a silicon wafer, and the wafer was slowly rotated. Using the previously determined friction coefficient, $\mu$, a measured angle at which the robot begins to slide on the wafer, $\alpha$, and a simple free body diagram, the magnitude of the adhesion force can be derived:

$$
F_{a d h}=m g\left(\frac{\sin (\alpha)}{\mu}-\cos (\alpha)\right)
$$

From Fig. 9, OPP velocities are higher at low adhesion because the in-plane coil is always on, allowing sliding 


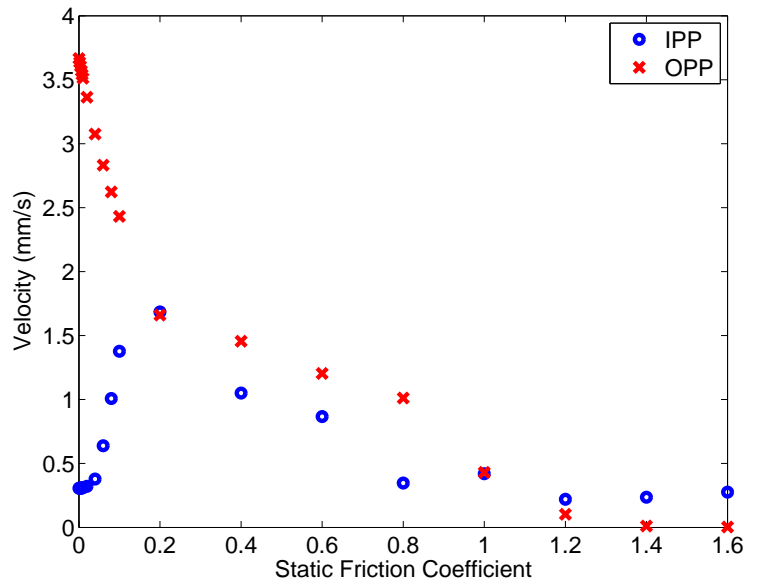

Fig. 8. Simulated effect of changing the static and kinetic friction coefficients on the steady state velocity of the robot. The kinetic friction coefficient is taken to be $70 \%$ of the static. Pulsing frequency is $100 \mathrm{~Hz}$, with a $2.5 \mathrm{mT}$ maximum pulsing magnetic field.

motion. As adhesion increases, OPP motion is influenced more than IPP for reasons similar to increasing friction: OPP translates the robot during upswing, when normal forces (which increase with adhesion) and friction forces are at their highest, whereas IPP translates the robot during the downswing, when the importance of these forces is minimized.

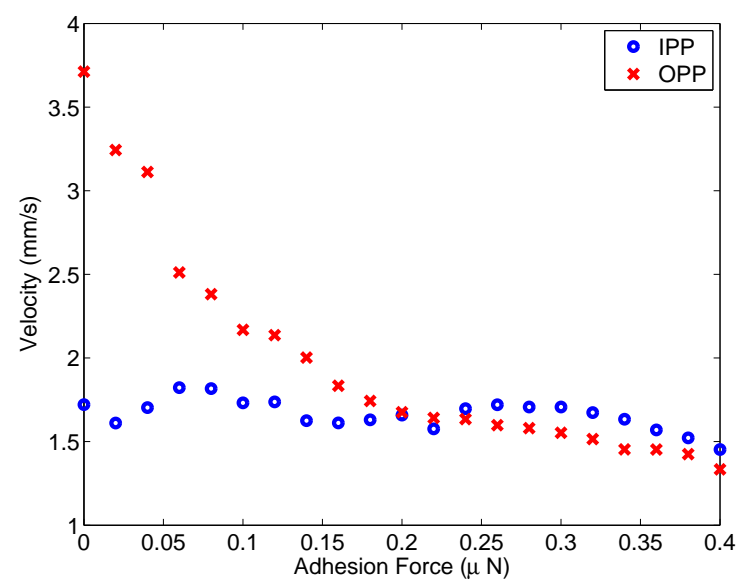

Fig. 9. Simulated effect of changing the adhesive force, $F_{a d h}$, on the steady state velocity of the robot. Pulsing frequency is $100 \mathrm{~Hz}$, with a 2.5 $\mathrm{mT}$ maximum pulsing magnetic field.

\section{Damping}

Linear damping forces, $L_{x}, L_{z}$, and rotational damping torque $D_{y}$, are a result of fluid drag effects through a liquid layer that is assumed to form between the robot and the silicon surface. For modeling, linear damping forces are first estimated using a Couette flow fluid drag model:

$$
L_{x, z} \approx \frac{A_{b} \mu_{d}}{g_{0}} \times\{\dot{x}, \dot{z}\}[N]
$$

where $A_{b}$ is the area of the bottom of the robot, $\mu_{d}$ is the dynamic viscosity of the liquid layer, and $g_{0}$ is the separation from the surface, assumed to be $1.5 \mu \mathrm{m}$ from measurements of the surface roughness. This results in a linear drag $L_{x, z} \approx$ $2.4 \times 10^{-5} \times\{\dot{x}, \dot{z}\}[N]$. In a similar fashion, torque damping forces are estimated using the integral of a viscous drag force equation:

$$
D_{y}=C_{D} \frac{1}{2} \rho_{w} \int_{0}^{L / 2} W s^{3} d s \times \dot{\theta}^{2}[N \cdot m]
$$

where $C_{D} \approx \frac{19}{R e}$ is the constant drag coefficient at $R e \approx$ $\frac{L \dot{x}}{\nu}=0.22[20], \nu$ is the kinematic viscosity of water, $\rho_{w}$ is the density of water, and $s$ is a variable of integration. This results in a rotational damping torque $D_{y} \approx 6.7 \times 10^{-16} \times$ $\dot{\theta}^{2}[N \cdot m]$.

Both damping coefficients are later adjusted to match the simulation to experimental results. In reality, the damping forces will depend on the micro-robot's orientation angle, $\theta$. For purposes of simulation, estimated average damping coefficients are used to capture the overall behavior trends; in the future, angular-dependent drag coefficients may be used in simulation. The estimated constant damping forces used are:

$$
\begin{aligned}
& L_{x, z}=1.0 \times 10^{-5} \times\{\dot{x}, \dot{z}\}[N] \\
& D_{y}=9.0 \times 10^{-17} \times \dot{\theta}^{2}[N \cdot m]
\end{aligned}
$$

These damping terms are necessary to keep the simulated robot stable in both rotational and translational motion. With increasing linear damping, average micro-robot velocity decreases for both IPP and OPP control. Changes in the linear damping coefficient cause different behavior regimes to emerge. At very low values of linear damping, the velocity is controlled almost exclusives by friction forces. At higher values of linear damping, all motion is suppressed. Only in a very small range is micro-robot velocity controlled by linear damping. For lower values of rotational damping, there is little effect on micro-robot velocity. This is likely the case when rotational drag is much smaller than magnetic torque. As rotational damping increases, both OPP and IPP velocities decrease at about the same rate, supporting this theory.

\section{RESULTS AND DISCUSSION}

All testing was performed on the back side of a silicon wafer in open air. No special polishing or preparation was performed on the wafer. For both control methods, two different parameters were examined: (1) Maintaining a constant waveform pulsing frequency while varying the maximum voltage across the coils (as a result, varying the maximum magnetic field), and (2) maintaining a maximum voltage across the coils while varying the frequency of waveform pulsing.

For each experiment at each pulsing frequency, three trials were performed to attain an error estimate of the velocity. During the experiment, a video of the robot motion was recorded and post-processed. Two frames of the video, one near the beginning and one near the end of the robot's journey, were taken. In each position, the robot's central position was determined, and the total travel distance was 


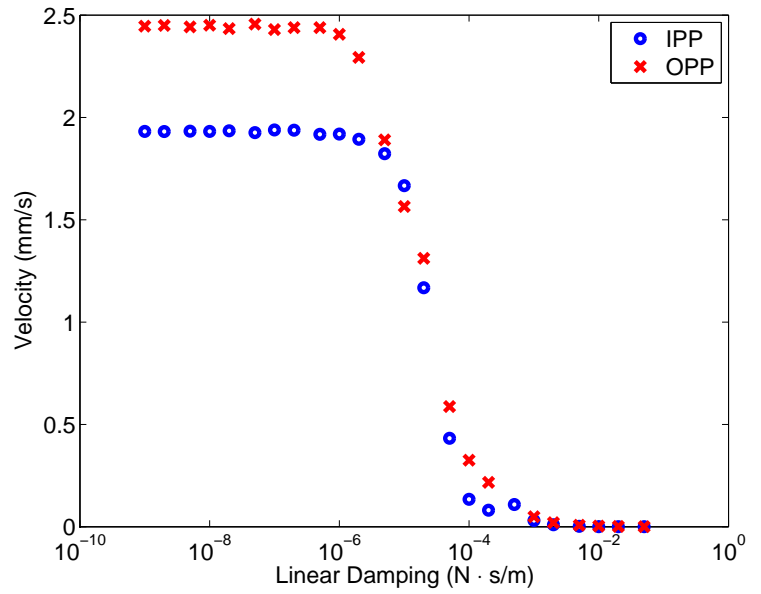

Fig. 10. Simulated effect of changing the linear damping on the steady state velocity of the robot. Pulsing frequency is $100 \mathrm{~Hz}$, with a $2.5 \mathrm{mT}$ maximum pulsing magnetic field.

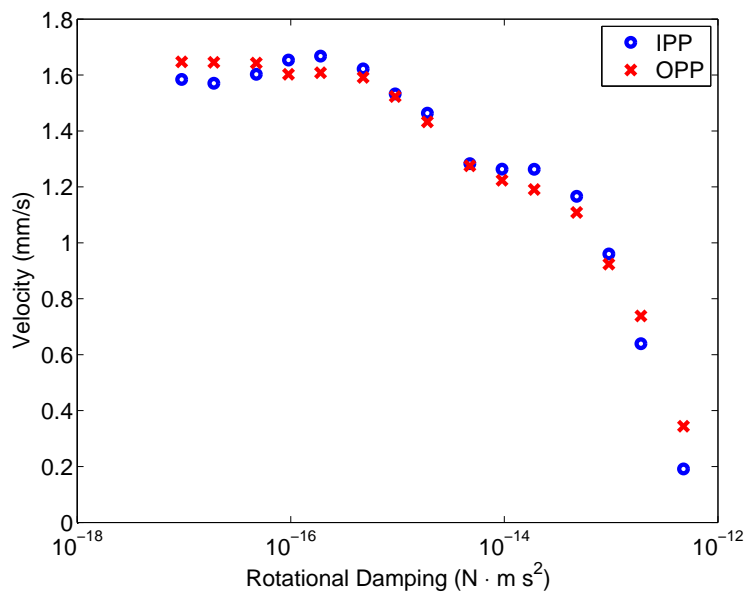

Fig. 11. Simulated effect of changing the rotational damping coefficient on the steady state velocity of the robot. Pulsing frequency is $100 \mathrm{~Hz}$, with a $2.5 \mathrm{mT}$ maximum pulsing magnetic field.

measured in pixels. A conversion ratio from the image to real-world distances in microns/pixel was empirically determined by counting the pixels across a known length. The total time for travel was also recorded to determine the velocity. Across a travel distance of about $5 \mathrm{~mm}$ with a positioning error of $1-2$ pixels (about $50 \mu \mathrm{m}$ ), results in a $1 \%$ error in measured distance.

For simulations, the average velocity was determined in a similar manner. The $x$-position and time was reported shortly after steady state motion was reached (after the third cyclic pulse), and also at a determined time signifying the end of the simulation. These two values were used to determine the average velocity of the simulated micro-robot.

Micro-robot velocity as a function of frequency for both IPP and OPP control appears to be linear for low frequencies in both experiment and simulation, as seen in Figs. 12 and 13. At higher frequencies, velocities appear to exhibit a slight

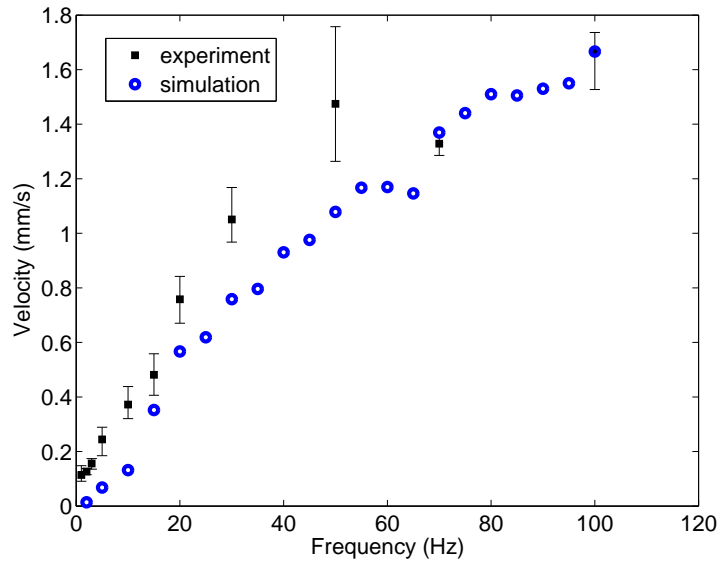

Fig. 12. Simulated and experimental robot velocity at varying frequencies, at a maximum pulsing magnetic field strength of $2.5 \mathrm{mT}$ under IPP control.

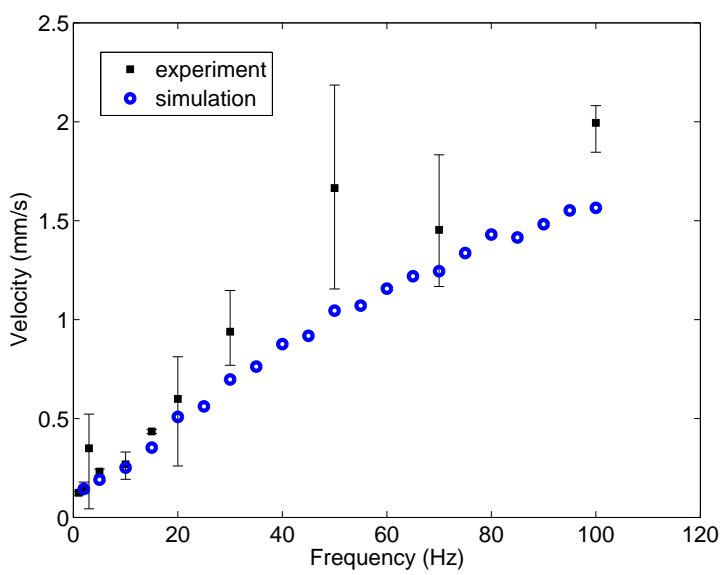

Fig. 13. Simulated and experimental robot velocity at varying frequencies, at a maximum pulsing magnetic field strength of $2.5 \mathrm{mT}$ under OPP control

roll-off in experiment. This roll-off may be due to the linear and rotational damping effects experienced by the microrobot.

Simulated results seem to underestimate micro-robot velocity for both IPP and OPP translation modes as a function of maximum field strength, shown in Figs. 14 and 15. For both IPP and OPP, the simulation suggests a linearly increasing dependence of velocity on field strength with some roll off for higher fields in IPP. This dependence is apparent in the IPP case, but is not as clear for OPP, which may be linearly increasing or may be relatively constant.

\section{CONCLUSION}

A detailed computer simulation that modeled the dynamics of a magnetically controlled micro-robot on a flat surface is proposed in this study. The parameters of the simulations were adjusted until an approximate match with reality was achieved. Both in simulation and in experiment, the magnetic micro-robot was subjected to alternating magnetic fields, which induced a stick-slip motion over the surface. The velocity of this motion as a function of both excitation 


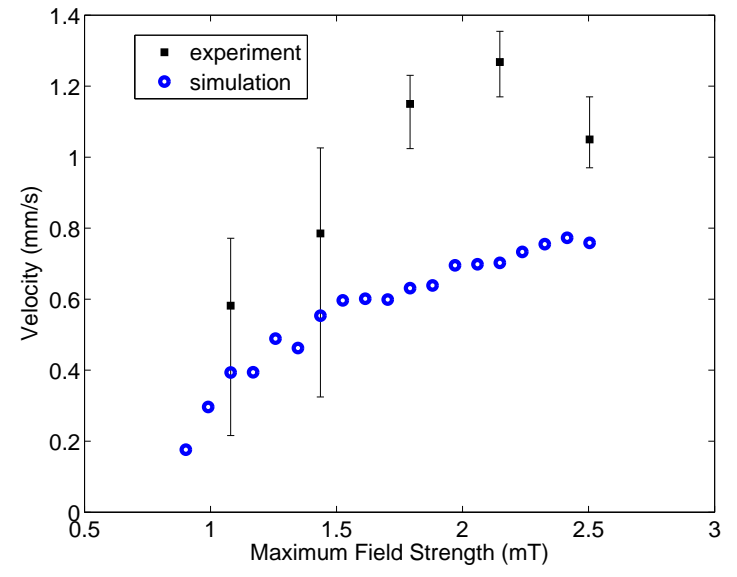

Fig. 14. Experimental robot velocity at varying maximum coil voltages (displayed as maximum field strength) at a constant pulse frequency of 30 $\mathrm{Hz}$ for IPP control.

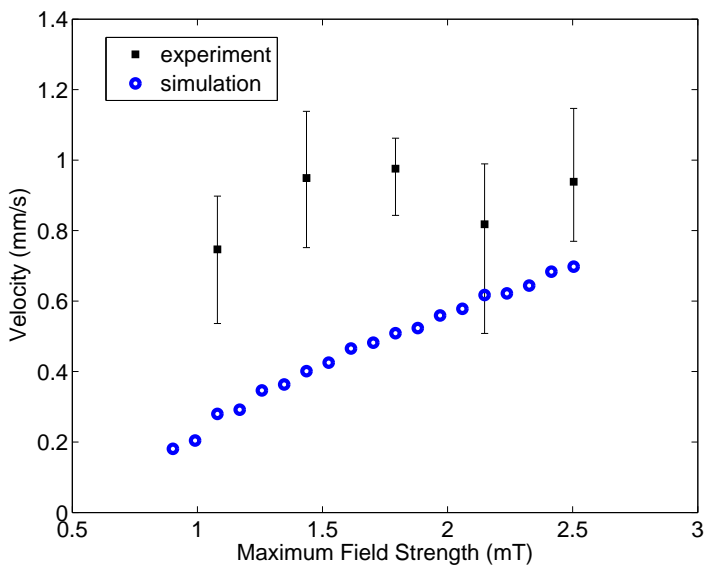

Fig. 15. Experimental robot velocity at varying maximum coil voltages (displayed as maximum field strength) at a constant pulse frequency of 30 $\mathrm{Hz}$ for OPP control.

frequency and maximum magnetic field for two different control methods were recorded and analyzed.

It was found that in both control methods, IPP and OPP control, the robot first attains a steady state angle with respect to the surface. This angle is much larger for IPP than for OPP. When one of the magnetic fields begins to oscillate, the robot will rock upward and downward. For IPP control, the robot will slide over the surface each time it rocks downward. Alternatively, in OPP control, the robot will slide each time it rocks upward. These behaviors were observed experimentally and accurately reproduced in simulation.

The dependence of robot velocity on friction, adhesion, linear damping, and rotational damping was explored in simulation. In addition, both frequency and peak voltage of OPP and IPP control were varied in simulation and experiment to determine their effects on robot velocity. The results obtained in simulation show general agreement with experiments.

Future work will include adapting the system for both coarse and fine motion control by using the simulation to refine the control signals and determine appropriate control laws in each case. In addition, vision algorithms are being developed for closed-loop computer control of the microrobot. Possible applications in micro-object manipulation, underwater control, and cooperative robotics are currently being explored.

\section{ACKNOWLEDGMENT}

The authors would like to thank L. Weiss and the Pittsburgh Infrastructure Technology Alliance for the use of the high-speed camera, and the NanoRobotics Laboratory members for all of their support and suggestions.

\section{REFERENCES}

[1] T. Ebefors et. al., "A Walking Silicon Micro-Robot," The 10th In Conference on Solid-State Sensors and Actuators (Transducers99), Sendai, Japan, pp 1202-1205. 1999.

[2] P. E. Kladitis, V. M. Bright, "Prototype Microrobots for Micropositioning and Micro-unmanned Vehicles," Sensors and Actuators, Vol. 80, No. 2, pp. 132-137, 2000.

[3] M. Mohebbi et. al., "Omnidirectional Walking Microrobot Realized by Thermal Microactuator Arrays," Proc. ASME Int. Mechanical Engineering Congress and Exposition, pp. 1-7, 2001.

[4] S. Martel, C. Tremblay, S. Ngakeng, and G. Langlois, "Controlled manipulation and actuation of micro-objects with magnetotactic bacteria,' Applied Physics Letters, Vol. 89, No. 233904, 2006.

[5] A. Yamazaki et. al., "Wireless micro swimming machine with magnetic thin film," Journal of Magnetism and Magnetic Materials, Vol. 272276 pp. e1741-e1742, 2004.

[6] R. Dreyfus et. al., "Microscopic artificial swimmers," Nature, Vol. 437, pp. 862-865, 2005

[7] K. Yesin, K. Vollmers, and B. Nelson, "Modeling and Control of Untethered Biomicrorobots in a Fluidic Environment Using Electromagnetic Fields," International Journal of Robotics Research, Vol. 25, pp. 527-536, 2006

[8] S. Martel et. al., "Three-Legged Wireless Miniature Robots for MassScale Operations at the Sub-Atomic Scale," Proceedings of the 2001 IEEE International Conference on Robotics and Automation, Seoul, Korea, pp. 3423- 3428, 2001.

[9] B. Donald et. al., "An Untethered, Electrostatic, Globally Controllable MEMS Micro-Robot,” Journal of Microelectromechanical Systems, Vol. 15, No. 1, pp. 1-15, 2006

[10] S. Martel, "Special surface for power delivery to wireless microelectro-mechanical systems," J. Micromech Microeng., 15, pp. S251258, 2005.

[11] K. Vollmers et. al., "Wireless Resonant Magnetic Microactuator for Untethered Mobile Microrobots," Applied Physics Letters, Vol. 92, No. $144103,2008$.

[12] O. Sul et. al., "Thermally Actuated Untethered Impact-driven Locomotive Microdevices," Applied Physics Letters, Vol. 89, No. 203512, 2006

[13] S. Floyd, C. Pawashe, and M. Sitti, "An Untethered Magnetically Actuated Micro-Robot Capable of Motion on Arbitrary Surfaces,' Proceedings of the 2008 IEEE International Conference on Robotics and Automation, Pasadena, CA, In Press.

[14] D. K. Cheng, "Field and Wave Electromagnetics, 2nd Edition," Addison-Wesley Publishing Company, Inc., 1992.

[15] W. Frix, G. Karady, and B. Venetz, "Comparison of Calibration Systems for Magnetic Field Measurement Equipment," IEEE Transactions on Power Delivery, Vol. 9, pp. 100-108, 1994.

[16] T. Boyer, "The Force on a Magnetic Dipole," Am. J. Phys. Vol. 56, Issue 8, pp. 688-692, 1987.

[17] Available Online: http://nanolab.me.cmu.edu/

[18] D. Stewart, "Finite-Dimensional Contact Mechanics," Philosophical Transactions: Mathematical, Physical, and Engineering Sciences, 359 (1789), Non-Smooth Mechanics, pp. 2467-2482, 2001.

[19] J. Israelachvili, "Intermolecular and Surface Forces," Academic Press, London, 1992

[20] B. Munson, D. Young, and T. Okiishi, "Fundamentals of Fluid Mechanics, 4th Edition,” John Wiley and Sons, Inc., 2002. 